\title{
Monitoring System Design for XLPE Power Cable Faults Based on Virtual
}

\section{Instruments}

\author{
Lai $\operatorname{Dian}^{1, a}$ \\ ${ }^{1}$ North China Electric Power University, 071006
}

Key Words: Power Transmission Lines, Power Cables, Fault Monitoring, Virtual Instruments

\begin{abstract}
With the vigorous development of power transmission project, the security and stability of power transmission lines appear to be more important than ever before. As one of the most significant devices in power transmission lines, the security guarantee of power cables is of great importance. Therefore, as is shown in this paper, a monitoring system for XLPE power cable faults that consists of a hybrid sensor, a signal processor and a monitor based on virtual instruments has been designed, which can be quite effective in electric power overhaul.
\end{abstract}

\section{Introduction}

With the rapid development of Chinese economy and urban construction, the power load has also increased. Currently, there is an increasing proportion of power cables in the power transmission and distribution system. Thanks to its excellent performance in insulativity, thermal and mechanical properties, and reliability of power supply, the XLPE power cable has enjoyed a rapid development since the 1960s. Now it has been applied to power transmission lines and power distribution networks under different voltages in the power system. What's more, it has even been used in some fields under high or super high voltage. Although there is a low probability for the cable lines to break down, the fault types can be various, such as the damage on cable insulators because of external force, discharging on the surface of electric cable accessories and aging of cable insulators. All these can lead to the break-down of cable lines. And once it happens, the power transmission will be disturbed and the power supply interrupted. Therefore, studies on the online monitoring technology of cable insulators have been quite popular among the scholars nowadays. To ensure a secure and stable operation of power cables, a real-time monitoring of how the cable insulator works should be done. In this way, people can predict the occurrence of faults and take appropriate measures such as overhauling or replacing the cables in advance.

In this paper, a hybrid sensor that can monitor partial discharging signals and ground circulation, a signal processor and a monitor based on virtual instruments have been designed. In this way, such faults as partial discharging and multi-point grounding can be monitored and analyzed, which can offer an effective guarantee for the secure power transmission of power cables.

\section{An analysis on the reasons and types of power cable fault}

The common power cable faults can be categorized into the following types:

(1) Mechanical damage: many faults are directly caused by the mechanical damage during the cable-laying process or other work near the cable lines after the installation.

(2) Aging and deterioration of cable insulators: the insulativity performance can be damaged when the air gap between the insulating mediums of cables gets free under the electric field. When the ionization of 
insulating mediums takes place, the ozone produced in the air gap will corrupt the insulator; and water in the insulator will also get the insulating fiber hydrolyzed, resulting in a reduction of insulativity.

(3) Chemical corruption: if the cable lines are set in areas where people work with acid-bases or there is a gas station nearby, the acid-bases and the benzene vapor will cause a large-sized and long-distanced corruption in the cable armor and sheath of lead or aluminum. Consequently, pits, cracks and holes will quickly follow, leading to power cable faults.

(4) Overvoltage: the overvoltage here mainly refers to the atmospheric overvoltage (namely, lightening) and the overvolatge inside the cables. As is shown in the analysis of actual faults, many of the outside terminal faults are caused by the atmospheric overvoltage. In addition, defects of the cable itself can also lead to faults under the circumstance of atmospheric overvoltage.

The above-mentioned faults mainly appear to be partial discharging and multi-point grounding. Therefore, this paper is aimed to monitor and analyze the ground circulation and pulse current signals of partial discharge.

\section{Structure and function design of the monitoring system}

The designed monitoring system for XLPE power cables faults based on virtual instruments in this paper includes a hybrid sensor which can monitor ground circulation and pulse signals of partial discharge, a signal processor and a monitor based on virtual instruments.

If the metal sheath of cables connect to the ground at many points during the operation, there will be a current circulation running in the sheath. And this will cause an increase in the cable heat, accelerate the aging of cables and eventually result in cable operation accidents. The increased current circulation in the condition of multi-point grounding will trigger partial discharge and send out pulse current signals. Hence, a hybrid sensor can be designed so as to monitor pulse current signals and multi-point grounding signals at the same time. The Rogowski coil has been used in the designed hybrid sensor in this paper. And a current transformer based on Rogowski coil has been used in this paper as the sensor unit of primary current. Because the secondary output voltage signals of the Rogowski coil are the differential of primary current, to gain signals which match the primary current in direct proportion, there is a necessity to do an integral processing with the output current signals of Rogowski coil. In this way, the appropriate signals can be obtained. The design principle is shown as follows:

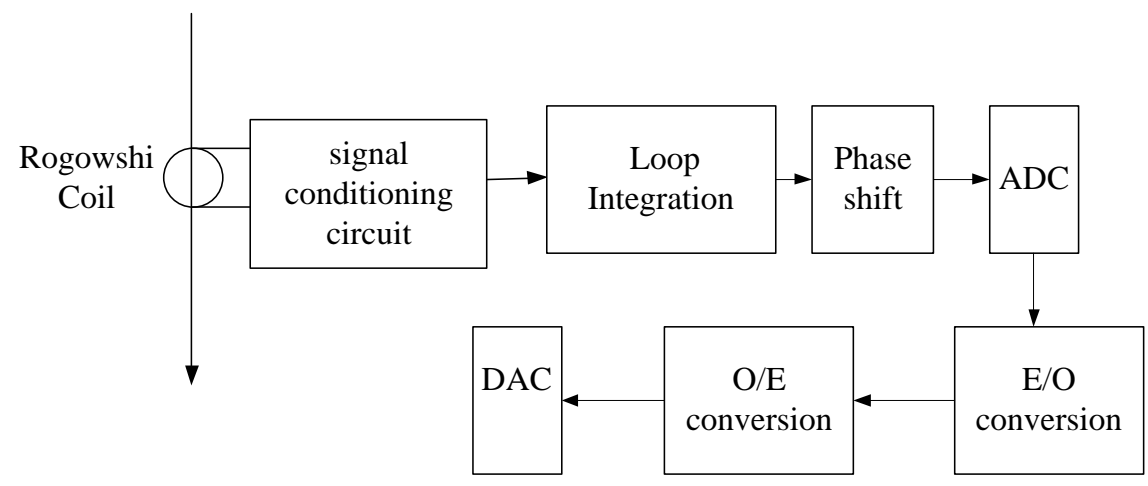

Fig.1 Design of Sensor

Rogowshi coil, Signal conditioning circuit, Integral circuit, Phase shift circuit, Analog-to-digital 
conversion,E/O switching circuit, $\mathrm{O} / \mathrm{E}$ switching circuit, D/A switching circuit

The signal frequency rand of the designed sensor in this paper ranges from 0 to $30 \mathrm{MHZ}$. Once transmitted into the signal processor, the collected signals will go through the smoothing module, signal isolation and amplifier module, and eventually get to the signal separation unit. Through the frequency demultiplication technology, the received signals will be divided into low frequency signals of 0-1 MHZ and high frequency signals of 1-30 MHZ. And signals of these two frequency brand will then be output through different accesses.

Generally speaking, the monitoring host consists of a PC server (the main frequency of CPU: 3.6 Ghz, RAM: 4G, Memory: 500G) and data-reading modules. The Linghua data collection card 9812 has bee used in the data-reading module in this paper. And in this module, signals will be collected, data will be processed and then transmitted to the industrial personal computer.

\section{Upper computer design}

To achieve a better monitoring of partial discharging signals and ground circulation of power cables, and to gain a better design of monitoring system with a combination of virtual instrument technology, LABVIEW has been employed here to design the system since it owns such advantages as easy operation and algorithm implementation. To meet the demand on system function, the LABVIEW 2014 of the NI company has been adopted as the software development platform in the monitoring system. And the surface is shown as follows:

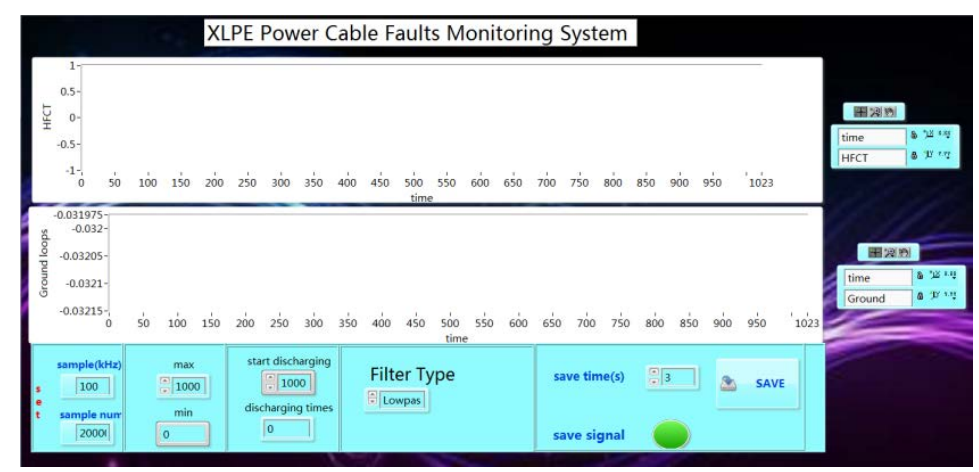

Fig.2 Monitoring surface of upper computer

\section{Conclusion}

In this paper, a hybrid sensor that can monitor partial discharging signals and ground circulation, a signal processor and a monitor based on virtual instruments have been designed. In this way, faults such as partial discharging and multi-point grounding can be detected and analyzed, so that people can be alert to the fault signals and call the police in time.

\section{References:}

[1]Zhu Bo. A Study on the Online Monitoring and Faults Locating Technology of Power Cable Insulator in Long Distance [D]. Harbin University of Science and Technology, 2015

[2]Sun Hongmei. Research and Development of Fault Online Monitoring System of XLPE Power Cable Insulator [D]. Hunan University,2004

[3]Ren Yanxia. Diagnose and Detection of Power Cable Faults [D]. Beijing Jiaotong University, 2008

[4]Cui Jinjun. Research on Power Cable Fault Location Methodology and Realization of Online 
Detection Device [D]. North China Electric Power University, 2015

[5] Bao Yongsheng. Online Monitoring and Faults Diagnose of Partial Discharge in Power Cables [D]. Beijing Jiaotong University,2012

[6] Zhang Cun. Research and Design of Online Monitoring System of Power Cables [D]. University of Electronic Science and Technology of China,2012.

[7] Duan Jinying. Onlie Monitoring System of Traveling Wave of Cable Faults Based on LabVIEW [J]. Instrument Technique and Sensor, 2011, 12:43-45

[8] Liao Yuanzhong. Research on Online Monitoring System of XLPE Cable Insulator Faults [J]. Guangxi Electric Utility, 2008, 04:117-120 